\title{
A Clinical Informatics Program Directors' Proposal to the American Board of Preventive Medicine
}

\author{
Natalie M. Pageler ${ }^{1}$ Peter L. Elkin²,3,4 Joseph Kannry ${ }^{5}$ Michael G. Leu ${ }^{6}$ Bruce Levy $^{7}$ \\ Christoph U. Lehmann 8
}

${ }^{1}$ Clinical Informatics Fellowship, Stanford School of Medicine, Palo
Alto, California, United States
${ }^{2}$ Department of Biomedical Informatics, Jacobs School of Medicine and
Biomedical Sciences, University at Buffalo, Buffalo, New York, United States
${ }^{3}$ Department of Veterans Affairs, VA Western New York Healthcare
System, Buffalo, New York, United States
${ }^{4}$ Faculty of Engineering, University of Southern Denmark, Denmark
${ }^{5}$ Clinical Informatics Fellowship, Icahn School of Medicine at Mount
Sinai, New York, New York, United States
${ }^{6}$ Departments of Pediatrics and Biomedical Informatics and Medical
Education, University of Washington, Seattle, Washington, United States
${ }^{7}$ Geisinger Commonwealth School of Medicine, Scranton,
Pennsylvania, United States
${ }^{8}$ Clinical Informatics Center, UT Southwestern, Dallas, Texas, United States

Address for correspondence Christoph U. Lehmann, MD, 1918 Olive Street, \#302, Dallas, TX 75201, United States

(e-mail: christoph.lehmann@utsouthwestern.edu).

Appl Clin Inform 2020;11:483-486.

\section{Abstract}

Keywords

- clinical informatics

- professional training

- education

- physician

- general healthcare providers

- training and education requirements

- health care

- accreditation and licensure

- clinical informatics fellowship
In 2013, the American Board of Preventive Medicine (ABPM) and the American Board of Pathology (ABPath) offered the first board certification examination in Clinical Informatics to eligible physicians in the United States. In 2022, the Practice Pathway will expire and in 2023 only candidates eligible through the Fellowship Pathway will be eligible for the board certification. To date, Clinical Informatics as a specialty has not had a regular match process and used a controlled offer-acceptance process that does not meet candidates' or programs' needs. Fellows may not be offered a position with their top choice program initially, and they may accept offers from other programs to avoid risk by ensuring that they have a fellowship position. Programs have to consider losing an applicant in the first round in the ranking of applicants. The process is open to manipulation including early agreements between program directors and candidates. In this open letter to the ABPM, program directors make the case for a third-party match and are calling on the ABPM to leverage its status as the Clinical Informatics certifying body and its existing infrastructure to implement a Clinical Informatics match. received

May 27, 2020

accepted after revision

June 28,2020 (c) 2020 Georg Thieme Verlag KG Stuttgart · New York
DOI https://doi.org/

10.1055/s-0040-1714348.

ISSN 1869-0327. 


\section{Background and Significance}

\section{The Subspecialty}

In 2013, the American Board of Preventive Medicine (ABPM) and the American Board of Pathology (ABPath) offered the first board certification examination in Clinical Informatics to eligible physicians in the United States. ${ }^{1-3}$ Consistent with American Board of Medical Specialties (ABMS)-approved practices for new subspecialties, a Practice Pathway was initially approved for 5 years and was extended for an additional 5-year period after the ABMS approved an ABPM petition to extend the timeline. Thus, applications for board certification in Clinical Informatics via the Practice Pathway will be accepted only through the 2022 application cycle. Beginning in 2023, the only path to board eligibility will be through the Fellowship Pathway. ${ }^{4}$

The Fellowship Pathway for Clinical Informatics Board Certification requires the physician to have graduated from a medical school meeting ABPM/ABPath standards, to hold an active primary specialty certification from an ABMS Member Board, to have an unrestricted license to practice medicine in every state or territory in which the physician has a license to practice medicine, to provide a letter of reference from an ABMS-certified physician, and to demonstrate the successful completion of a 24-month full-time Accreditation Council for Graduate Medical Education (ACGME)-accredited Clinical Informatics fellowship. ${ }^{5}$

In 2017, there were 18 approved Fellowship Pathway applicants, and 222 approved Practice Pathway applicants for CI board certification. ${ }^{4}$ Based on a 2017 survey of Clinical Informatics fellowship applicants for the 2016-2018 and 2017-2019 cohorts, the total number of fellowship applicants was estimated to be between 42 and 69 for the 2016-2018 cohort (applying to 24 positions) and between 52 and 85 for the 2017-2019 cohort (applying to 30 positions). Candidates, who succeeded in obtaining a position, applied to more programs, with averages of 4.2 and 5.5 programs per applicant, respectively. ${ }^{6}$

Since then, even with continued challenges to finance Clinical Informatics fellows, ${ }^{7}$ the number of Clinical Informatics fellowship programs (and thus, number of positions, resulting in potential Fellowship Pathway candidates) continues to grow rapidly. We anticipate the number of Clinical Informatics fellowship applicants will rise as the Practice Pathway is discontinued. As of June 2020, there are currently 46 accredited programs. By 2023, we anticipate the number of applicants to exceed 100 annually.

\section{The "Match" to Date}

To date, Clinical Informatics programs have agreed to a simultaneous offer process conducted under the leadership of Dr. Bruce Levy. In advance, programs announce the number of spots available for new fellows, and a matching document of open positions is created. On a mutually agreedupon time and date (the second Wednesday of December, at 12 PM Eastern/11 AM Central/10 AM Mountain/9 AM Pacific), program directors call the applicant at the top of their lists and offer a position. The applicant has an amount of time preagreed by the programs to make a decision (initially 2 hours, then 1 hour, and most recently 30 minutes). Program directors can only call applicants if there is an available position for that applicant at that time.

- If the applicant accepts the offer, his/her name is entered in a document available online only to program directors. Program directors update another spreadsheet available online to all applicants with the counts of total available and filled positions for the programs, so that the applicants can know if their program of choice has filled.

- If the applicant rejects the offer, the program director can call the next applicant on his/her list.

In 2019, for the 2020-2022 cohort, 41 applicants accepted offers through the simultaneous offer process to 25 programs.

\section{Challenges}

Despite much good will and very high participation by Clinical Informatics fellowships, the simultaneous offer process has had significant drawbacks. Unlike the National Resident Matching Program (NRMP) match, this "controlled scramble" is not optimized for fellow preferences. The simultaneous nature of the offers leads to blockages where applicants must decide whether to accept offers in hand or wait to see if a program that the applicant prefers and that has not filled all positions will call them. Applicants therefore may not accept offers with their top-choice program to avoid risk (especially if they see desirable programs filling up) and may instead accept the first offer they receive, as the programs may be deadlocked on the top applicants. Similarly, the process is not ideal for programs either, making it difficult for programs to rank the applicants without considering the likelihood of losing an applicant in the first round. Over time, with more programs and applicants applying to more programs, these blockages appear to become more severe and problematic. Furthermore, the process is open to manipulation including early agreements between program directors and applicants that are undetectable if the agreements are not announced until "match" day. The process lacks transparency and results are not easily reproducible.

\section{Proposing a Third-Party Match}

The community of Clinical Informatics program directors is proposing a third-party match as used in most other specialties and subspecialties.

\section{Benefits of a Third-Party Match program}

A third-party match program creates a neutral venue and an even playing field as all participants follow the same rules and adhere to the same deadlines. Applicants and programs can consider all their options before making decisions, and there may be less pressure on programs to extend early offers to get the best candidates.

Match participation makes the recruitment and appointment process easier and transparent. A match encourages participants to rank their top choices and will consistently 
yield the best possible outcome for participants, with a slight preference for the preferences of the fellow candidates over those of the fellowship programs.

\section{Why Not NRMP, SF Match, or others?}

The Clinical Informatics program director community reached out to third parties that administer matches. However, the specific needs inherent to the Clinical Informatics subspecialty have been obstacles for obtaining buy-in. Because Clinical Informatics programs are "housed" in various specialty departments, and may have different funding mechanisms for different slots in their programs, ${ }^{7}$ hosting programs may have specific requirements on the number of candidates with certain primary specialty board eligibility or certification complicating the matching algorithm. NRMP, San Francisco (SF) Match, and others declined to conduct the match for Clinical Informatics as they decided not to host a modified algorithm.

In response, under the leadership of Dr. Michael Leu, the community of Clinical Informatics program directors developed a prototype matching algorithm that meets the needs of the applicants and the programs, to be executed by a neutral third party. The algorithm does not require discretionary decisions by the executing third party and will run independently, minimizing effort or risk to the third party.

\section{Requirements}

To execute a match successfully, applicants and programs must register in a common system. In addition to the typical match inputs of rank order lists from the applicants and from the programs, the programs can also add "constraints" to the match (e.g., our program can accept one applicant from this type of applicants). For example, a program with one spot funded by Pediatrics could specify that it can take one applicant from all of their Pediatric applicants. The candidate's specialty would be known by the programs through application materials and interviews and would be populated to the match system when the candidate registers. At an agreedupon day, the matching algorithm would determine the program-candidate pairs and notify programs and candidates of the results.

The algorithm for the match is based upon the Nobel Prize winning work on the Gale-Shapley algorithm (also known as the deferred acceptance algorithm). This is the method that underpins the NRMP system and can find either the solution that is optimal for the participants on one side of the matching or for the participants on the other side. In our case, the algorithm has been optimized for the fellowship applicants taking into considerations the restrictions of programs. The algorithm has been modified to consider the constraints on acceptance, which is a function of slots, specialty, and funding at each site.

\section{Why ABPM?}

ABPM has a stake in supporting fellowship programs and encouraging applicants to select the subspecialty. The certification focus and history of the ABPM on preventive medicine, epidemiology, and public health align well with Clinical
Informatics focus on the delivery of evidence-based medical practice through electronic health records and clinical decision support with a focus on individual patient care and a population/community impact.

Currently most applicants for the Clinical Informatics Board Examination apply to the ABPM. Providing a match would increase the number of desired matches, and if as anticipated more matches result, this may increase the number of boardeligible providers. ABPM is already a known and trusted third party involved in the certification of board-eligible Clinical Informatics candidates. ABPM has the infrastructure and support structure to allow individuals and programs to register. The additional burden would be small and could be offset by charging programs and applicants for the service. This might also be strategically helpful if other specialties certified by ABPM would need assistance in constructing a match process in the future.

By providing a match program, ABPM will further leverage its status as the $\mathrm{CI}$ certifying body and bring more visibility to clinical informatics within formal medical organizations like ACGME, ABMS, Central Medical Services Society (CMSS), Association of American Medical Colleges (AAMC), and other professional organizations, which in turn may increase the number of interested applicants in the future.

\section{Costs}

We expect that the costs for conducting the match will be offset by the registration and site fees. ABPM would have to develop a website that allows registration of applicants and programs, and allows the entering of priority lists. The matching algorithm will be provided at no cost. Additional services will include e-mail/telephone support for programs and applicants with issues and will use the existing ABPM infrastructure.

In speaking with the American Urology Association (AUA), which provides the match for urology residents and fellows, the program director community learned that the AUA charges applicants a $\$ 200$ fee and programs a $\$ 325$ fee to manage the match. To date, AUA has reported no legal issues or challenges in the over 35 years that AUA has been hosting the match for 450 to 500 residency and 25 to 30 fellowship applicants annually. The support effort for programs and applicants is estimated at approximately 300 hours/year.

\section{Conclusion}

The Community of Clinical Informatics Program Directors is requesting that the American Board of Preventive Medicine explore the possibility of hosting the annual match for Clinical Informatics fellowship applicants. ABPM has a history of stepping up and supporting the Clinical Informatics community when Clinical Informatics was looking for a home for its board certification process. The Community of Clinical Informatics Program Directors believes that APBM (in a cost-neutral fashion) as a neutral third-party matching organization would greatly improve the process for applicants and programs, provide fairness, produce better matches, provide transparency to the process, and would increase the applicant pool for the Clinical Informatics Board Examination. 


\section{Multiple Choice Questions}

1. When initially approved as a new subspecialty, the Practice Pathway was available for how many years?
a. 10 years
b. 5 years
c. 2 years
d. 1 year

Correct Answer: The correct answer is option b, 5 years. Initially the Practice Pathway was available only for 5 years but a request to ABMS was approved to extend the period to a total of 10 years.

2. The benefits of a neutral third-party match include which of the following:
a. Varying deadlines
b. Opportunity to extend early offers
c. Fairness
d. Secret process

Correct Answer: The correct answer is option c, fairness. A third-party match provides neutral venue and an even playing field as all participants follow the same rules and adhere to the same deadlines. Applicants and programs can consider all their options before making decisions, and there is no pressure on programs to extend early offers to get the best applicants.

Protection of Human and Animal Subjects

No human and/or animal subjects were not included in the creation of this letter to the editor.

\section{Conflict of Interest}

C.U.L. reports support from the International Medical Informatics Association, Thieme, and Springer, outside the submitted work. M.G.L. reports working with a workgroup of program directors to define the requirements for the match, and to create an algorithm to implement the match. He intends to "donate" the algorithm to the host organization and not to profit from this algorithm. The other authors have nothing to report.

\section{References}

1 Lehmann CU, Shorte V, Gundlapalli AV. Clinical informatics subspecialty board certification. Pediatr Rev 2013;34(11):525-530

2 Detmer DE, Munger BS, Lehmann CU. Clinical informatics board certification: history, current status, and predicted impact on the clinical informatics workforce. Appl Clin Inform 2010;1(01):11-18

3 Gundlapalli AV, Gundlapalli AV, Greaves WW, et al. Clinical informatics board specialty certification for physicians: a global view. Stud Health Technol Inform 2015;216:501-505

4 Lehmann CU, Gundlapalli AV, Williamson JJ, et al. Five years of clinical informatics board certification for physicians in the United States of America. Yearb Med Inform 2018;27(01):237-242

5 Silverman H, Lehmann CU, Munger B. Milestones: critical elements in clinical informatics fellowship programs. Appl Clin Inform 2016;7(01):177-190

6 Bell DS, Baldwin K, Bell EJ III, et al. Characteristics of the national applicant pool for clinical informatics fellowships (2016-2017). AMIA Annu Symp Proc 2018;2018:225-231

7 Lehmann CU, Longhurst CA, Hersh W, et al. Clinical Informatics Fellowship Programs: in search of a viable financial model: an open letter to the Centers for Medicare and Medicaid Services. Appl Clin Inform 2015;6(02):267-270 